\title{
Global Exponential Robust Stability of Static Interval Neural Networks with Time Delay in the Leakage Term
}

\author{
Guiying Chen ${ }^{1,2}$ and Linshan Wang ${ }^{1}$ \\ ${ }^{1}$ School of Mathematical Science, Ocean University of China, Qingdao 266100, China \\ ${ }^{2}$ School of Mathematical Science, Liaocheng University, Liaocheng 252059, China \\ Correspondence should be addressed to Linshan Wang; wangls@ouc.edu.cn
}

Received 24 July 2013; Accepted 12 December 2013; Published 12 January 2014

Academic Editor: Subhas Abel

Copyright (c) 2014 G. Chen and L. Wang. This is an open access article distributed under the Creative Commons Attribution License, which permits unrestricted use, distribution, and reproduction in any medium, provided the original work is properly cited.

The stability of a class of static interval neural networks with time delay in the leakage term is investigated. By using the method of $M$-matrix and the technique of delay differential inequality, we obtain some sufficient conditions ensuring the global exponential robust stability of the networks. The results in this paper extend the corresponding conclusions without leakage delay. An example is given to illustrate the effectiveness of the obtained results.

\section{Introduction}

Recently, neural networks have been widely studied because of their successful applications in different areas, such as pattern recognition, image processing, detection of moving objects, and optimization problems. The stability of the neural networks with time delay, upon which these applications largely depend, has been extensively studied (see [1-10]). However, to the best of our knowledge, there has been very little existing work on neural networks, especially, on static neural networks with time delay in the leakage term [11-18]. This is due to some theoretical and technical difficulties [13]. So, the main purpose of this paper is to study the stability of the static interval neural networks with time delay in the leakage term. By using the properties of $M$-matrix and delay differential inequality, we obtain some sufficient conditions ensuring the global exponential robust stability. Our results extend the corresponding conclusions without leakage delay.

\section{Model Description and Preliminaries}

In this section, we list all the notations which will be frequently used throughout the paper and give a few definitions, lemmas, and assumptions.

Notations. Let $R$ be the set of real number, and let $R^{n}$ and $R^{m \times n}$ be the space of $n$-dimensional real vectors and $m \times n$ real matrices, separately. $E$ denotes an $n \times n$ unit matrix.
$N \triangleq\{1,2, \ldots, n\}$. For $A, B \in R^{m \times n}$ or $A, B \in R^{n}$, the notation $A \geq B(A>B)$ means that each pair of corresponding elements of $A$ and $B$ satisfies the inequality " $\geq(>)$." $|\cdot|$ denotes the Euclidean norm. For any $u \in R, \operatorname{sgn}(u)$ is the sign function of $u$.

$C[X, Y]$ denotes the space of continuous mappings from the topological space $X$ to the topological space $Y$. Particularly, let $C \triangleq C\left([-\tau, 0], R^{n}\right)$ denote the family of all continuous $R^{n}$-valued function $\phi$ defined on $[-\tau, 0]$ with the norm $\|\phi\|=$ $\sup _{-\tau \leq s \leq 0}|\phi(s)|$.

For $x \in R^{n}, \varphi \in C$, we define $[x]^{+}=\left(\left|x_{1}\right|, \ldots,\left|x_{n}\right|\right)^{T}$, $[\varphi(t)]_{\tau}=\left(\left[\varphi_{1}(t)\right]_{\tau}, \ldots,\left[\varphi_{n}(t)\right]_{\tau}\right)^{T},\left[\varphi_{i}(t)\right]_{\tau}=\sup _{-\tau \leq s \leq 0}\left\{\varphi_{i}(t+\right.$ $s)\}, i \in N$, and $[\varphi(t)]_{\tau}^{+} \triangleq\left[[\varphi(t)]^{+}\right]_{\tau} . D^{+} \varphi(t)$ denotes the upper-right-hand derivative of $\varphi(t)$ at time $t$.

Consider the following interval static neural network model with leakage delay:

$$
\begin{aligned}
\frac{d x_{i}(t)}{d t}=- & a_{i}(\lambda) x_{i}(t-\sigma) \\
+ & f_{i}\left(\sum_{j=1}^{n} \int_{-\tau(\lambda)}^{0} x_{j}(t+\theta) d w_{i j}(\theta, \lambda)+I_{i}\right), \\
t \geq 0, & \\
& x_{i}(t)=\phi_{i}(t), \quad-r \leq t \leq 0,
\end{aligned}
$$


where $i, j=1,2, \ldots, n, x_{i}$ and $I_{i}$ denote the state and the external inputs of the $i$ th neuron, separately. The integer $n$ corresponds to the number of units in a neural network, and $f_{i}(\cdot)$ denotes the signal propagation function of the $i$ th unit. $\lambda \in \Lambda \subset R$ is a parameter, and $a_{i}(\lambda)$ represents the rate with which ith neuron will reset its potential to the resting state in isolation when disconnected from the network and external inputs. $w_{i j}(\theta, \lambda)$ is nondecreasing bounded variation functions on $[-\tau(\lambda), 0]$, and $\int_{-\tau(\lambda)}^{0} x_{j}(t+\theta) d w_{i j}(\theta, \lambda)$ is a Lebesgue-Stieltjes integration. There exist positive constants $\tau, \underline{a}_{i}, \bar{a}_{i}$, and $w_{i j}^{*}$ such that for any $\lambda \in \Lambda, 0<\underline{a}_{i} \leq a_{i}(\lambda) \leq$ $\bar{a}_{i}, 0 \leq \tau(\lambda) \leq \tau$, and $\left|\int_{-\tau(\lambda)}^{0} d w_{i j}(\theta, \lambda)\right| \leq w_{i j}^{*}<\infty$. $\sigma \geq 0$ represents the leakage delay, $r=\max \{\sigma, \tau\} . \phi(s)=$ $\left(\phi_{1}(s), \ldots, \phi_{n}(s)\right)^{T}$, where $\phi_{i}(s)$ is derivative on $[-r, 0]$, and $\left[\dot{\phi}_{i}\right]_{r}^{+} \leq m_{i}, i \in N$.

Definition 1. The equilibrium point $x^{*}$ of system (1) is said to be globally exponentially stable if there exist a positive constant $\gamma$ and a vector $M>0$ such that

$$
\left[x(t)-x^{*}\right]^{+} \leq M e^{-\gamma\left(t-t_{0}\right)}, \quad t \geq t_{0} .
$$

Definition 2. System (1) is said to be globally exponentially robustly stable if its equilibrium point $x^{*}$ is globally exponentially stable for any $\tau(\lambda) \in[0, \tau]$ and $a_{i}(\lambda) \in\left[\underline{a}_{i}, \bar{a}_{i}\right]$.

Definition 3 (see [19]). Let the matrix $D=\left(d_{i j}\right)_{n \times n}$ with $d_{i i}>$ 0 and $d_{i j} \leq 0, i \neq j, i, j=1,2, \ldots, n$. Then each of the following conditions is equivalent to the statement " $D$ is a nonsingular M-matrix."

(1) All the leading principle minors of $D$ are positive.

(2) The diagonal elements of $D$ are all positive, and there exists a positive vector $d$ such that $D d>0$ or $D^{T} d>0$.

Lemma 4 (see [20]). Let $a<b \leq+\infty$, and $v(t) \epsilon$ $C\left[[a, b], R^{n}\right]$ satisfies

$$
\begin{gathered}
D^{+} v(t) \leq P v(t)+Q[v(t)]_{\tau}, \quad t \in[a, b), \\
v(a+s) \in P C, \quad s \in[-\tau, 0]
\end{gathered}
$$

where $P=\left(p_{i j}\right)_{n \times n}, p_{i j} \geq 0$ for $i \neq j, Q=\left(q_{i j}\right)_{n \times n} \geq 0$, and $J=\left(J_{1}, \ldots, J_{n}\right)^{T} \geq 0, i, j=1,2, \ldots, n$. Suppose that there exist a scalar $\lambda>0$ and a vector $z=\left(z_{1}, z_{2}, \ldots, z_{n}\right)^{T}>0$ such that

$$
\left[\lambda E+P+Q e^{\lambda \tau}\right] z<0 .
$$

If the initial condition satisfies

$$
\begin{array}{r}
v(t) \leq M z e^{-\lambda(t-a)}-(P+Q)^{-1} J, \\
M \geq 0, \quad t \in[a-\tau, a],
\end{array}
$$

then $v(t) \leq M z e^{-\lambda(t-a)}-(P+Q)^{-1} J$ for $t \in[a, b)$. tions.
$\left(\mathrm{A}_{1}\right)$ The signal propagation functions $f_{i}(\cdot)$ are Lipschitz continuous; that is, there are positive constants $k_{i}$, $i \in N$ such that for all $s_{1}, s_{2} \in R$

$$
\left|f_{i}\left(s_{1}\right)-f_{i}\left(s_{2}\right)\right| \leq k_{i}\left|s_{1}-s_{2}\right| \text {. }
$$

$\left(\mathrm{A}_{2}\right)$ Let $-(U+V)$ be a nonsingular $M$-matrix, where

$$
\begin{gathered}
U=\left(u_{i j}\right)_{n \times n}, \quad V=\left(v_{i j}\right)_{n \times n}, \\
u_{i j}=0, \quad i \neq j, \quad u_{i i}=-\underline{a}_{i}, \\
v_{i i}=\sigma \bar{a}_{i}^{2}+\left(1+\sigma \bar{a}_{i}\right) k_{i} w_{i i}^{*}, \\
v_{i j}=\left(1+\sigma \bar{a}_{i}\right) k_{i} w_{i j}^{*}, \quad i \neq j .
\end{gathered}
$$

\section{Main Results}

Theorem 5. Suppose that the conditions $\left(A_{1}\right)$ and $\left(A_{2}\right)$ hold, and then system (1) has at least one equilibrium point.

Proof. From $\left(\mathrm{A}_{2}\right)$ and Definition 3, we know there exists a positive vector $d=\left(d_{1}, \ldots, d_{n}\right)^{T}$ such that $(U+V) d<0$. That is

$$
\begin{array}{r}
-\underline{a}_{i} d_{i}+\sigma \bar{a}_{i}^{2} d_{i}+\left(1+\sigma \bar{a}_{i}\right) k_{i} \sum_{j=1}^{n} w_{i j}^{*} d_{j}<0, \\
i=1, \ldots, n .
\end{array}
$$

From (8) we can get

$$
\begin{array}{r}
k_{i} \sum_{j=1}^{n} w_{i j}^{*} d_{j}<\frac{\underline{a}_{i}-\sigma \bar{a}_{i}^{2}}{1+\sigma \bar{a}_{i}} d_{i} \leq \underline{a}_{i} d_{i}, \\
i=1, \ldots, n .
\end{array}
$$

Combining with Definition 3, we know $\underline{A}-K W$ is a nonsingular $M$-matrix, where $\underline{A}=\operatorname{diag}\left(\underline{a}_{1}, \ldots, \underline{a}_{n}\right), K=$ $\operatorname{diag}\left(k_{1}, \ldots, k_{n}\right)$, and $W=\left(w_{i j}^{*}\right)_{n \times n}$.

In a similar way of proof for the literature [7], by the theory of topological degree and homotopy invariance theorem, the existence of the equilibrium point of system (1) can be proved. Suppose that $x^{*}$ is an equilibrium point of system (1), let $y_{i}(t)=x_{i}(t)-x_{i}^{*}, \varphi_{i}(t)=\phi_{i}(t)-x_{i}^{*}$, and then system (1) becomes

$$
\begin{aligned}
\frac{d y_{i}(t)}{d t}= & -a_{i}(\lambda) y_{i}(t-\sigma) \\
& +f_{i}\left(\sum_{j=1}^{n} \int_{-\tau(\lambda)}^{0}\left(y_{j}(t+\theta)+x_{j}^{*}\right) d w_{i j}(\theta, \lambda)+I_{i}\right) \\
& -f_{i}\left(\sum_{j=1}^{n} \int_{-\tau(\lambda)}^{0} x_{j}^{*} d w_{i j}(\theta, \lambda)+I_{i}\right), \quad t \geq 0, \\
& y_{i}(t)=\varphi_{i}(t), \quad-r \leq t \leq 0 .
\end{aligned}
$$


It is clear that the stability of zero solution to system (10) is equivalent to the stability of the equilibrium point $x^{*}$ of system (1). So we only consider the stability of zero solution to system (10).

Theorem 6. Assume that the conditions $\left(A_{1}\right)$ and $\left(A_{2}\right)$ are satisfied, and then the zero solution to system (10) is globally exponentially robustly stable.

Proof. From the Middle Value theorem, we obtain

$$
-y_{i}(t-\sigma)=-y_{i}(t)+\sigma \dot{y}_{i}(t-(1-\alpha) \sigma), \quad i=1,2, \ldots, n,
$$

where $0<\alpha<1$. Then from (10) we get

$$
\begin{aligned}
\frac{d y_{i}(t)}{d t}= & -a_{i}(\lambda) y_{i}(t) \\
& +\sigma a_{i}(\lambda) \dot{y}_{i}(t-(1-\alpha) \sigma) \\
& +f_{i}\left(\sum_{j=1}^{n} \int_{-\tau(\lambda)}^{0}\left(y_{j}(t+\theta)+x_{j}^{*}\right) d w_{i j}(\theta, \lambda)+I_{i}\right) \\
& -f_{i}\left(\sum_{j=1}^{n} \int_{-\tau(\lambda)}^{0} x_{j}^{*} d w_{i j}(\theta, \lambda)+I_{i}\right) .
\end{aligned}
$$

Case 1. If $0 \leq t<(1-\alpha) \sigma$, then $\dot{y}_{i}(t-(1-\alpha) \sigma)=\dot{\varphi}_{i}(t-(1-\alpha) \sigma)$.

Let $V_{i}(t)=\left|y_{i}(t)\right|, i \in N$. Then from $\left(\mathrm{A}_{1}\right)$, we have

$$
\begin{aligned}
& D^{+} V_{i}(t) \\
& =\operatorname{sgn}\left(y_{i}(t)\right) \dot{y}_{i}(t) \\
& =\operatorname{sgn}\left(y_{i}(t)\right)\left(-a_{i}(\lambda) y_{i}(t)+\sigma a_{i}(\lambda) \dot{y}_{i}(t-(1-\alpha) \sigma)\right. \\
& +f_{i}\left(\sum_{j=1}^{n} \int_{-\tau(\lambda)}^{0}\left(y_{j}(t+\theta)+x_{j}^{*}\right) d w_{i j}(\theta, \lambda)\right. \\
& \left.+I_{i}\right) \\
& \left.\quad-f_{i}\left(\sum_{j=1}^{n} \int_{-\tau(\lambda)}^{0} x_{j}^{*} d w_{i j}(\theta, \lambda)+I_{i}\right)\right) \\
& \quad+a_{i}(\lambda)\left|y_{i}(t)\right|+\sigma a_{i}(\lambda)\left|\dot{y}_{i}(t-(1-\alpha) \sigma)\right| \\
& \quad \sum_{j=1}^{n} \int_{-\tau(\lambda)}^{0}\left|y_{j}(t+\theta)\right| d w_{i j}(\theta, \lambda)
\end{aligned}
$$

$$
\begin{aligned}
& \leq-\underline{a}_{i}\left|y_{i}(t)\right|+\sigma \bar{a}_{i} m_{i}+k_{i} \sum_{j=1}^{n} w_{i j}^{*}\left[y_{j}(t)\right]_{\tau}^{+} \\
& =\sum_{j=1}^{n} p_{i j} V_{j}(t)+\sum_{j=1}^{n} q_{i j}\left[V_{j}(t)\right]_{\tau}+l_{i},
\end{aligned}
$$

where $p_{i i}=-\underline{a}_{i}, p_{i j}=0, i \neq j, q_{i j}=k_{i} w_{i j}^{*}$, and $l_{i}=\sigma \bar{a}_{i} m_{i}$.

From $\left(\mathrm{A}_{2}\right)$ and Definition 3, we know that there exists a vector $d>0$ such that $(U+V) d<0$. That is

$$
-\underline{a}_{i} d_{i}+\sigma \bar{a}_{i} d_{i}+\left(1+\sigma \bar{a}_{i}\right) k_{i} \sum_{j=1}^{n} w_{i j}^{*} d_{j}<0 .
$$

From (14), we can get

$$
\sum_{j=1}^{n} w_{i j}^{*} d_{j} \leq d_{i} \frac{a_{i}-\sigma \bar{a}_{i}^{2}}{1+\sigma \bar{a}_{i}} \leq d_{i} \underline{a}_{i} .
$$

Hence, $-(P+Q)$ is a nonsingular $M$-matrix. Thus, there exists a vector $z_{0}>0$ such that $(P+Q) z_{0}<0$. By using the continuity, we know there exists at least one constant $\lambda_{0}>0$ such that

$$
\left(\lambda_{0} E+P+Q e^{\lambda_{0} \tau}\right) z_{0}<0 .
$$

Since $\varphi \in C\left[[-r, 0], R^{n}\right]$, then, for $z_{0}>0$, there exists a constant $M \geq 0$ such that

$$
\begin{array}{r}
v(t)=[y(t)]^{+} \leq M z_{0} e^{-\lambda_{0} t}-(P+Q)^{-1} L, \\
t \in[-r, 0],
\end{array}
$$

where $L=\left(l_{1}, \ldots, l_{n}\right)^{T}$. Then from (13), (16), (17), and Lemma 4 , we get

$$
\begin{array}{r}
v(t)=[y(t)]^{+} \leq M z_{0} e^{-\lambda_{0} t}-(P+Q)^{-1} L, \\
t \in[0,(1-\alpha) \sigma] .
\end{array}
$$

Case 2. If $t \geq(1-\alpha) \sigma$, then from (10) we have

$$
\begin{aligned}
\dot{y}_{i}(t- & (1-\alpha) \sigma) \\
= & -a_{i}(\lambda) y_{i}(t-(2-\alpha) \sigma) \\
& +f_{i}\left(\sum _ { j = 1 } ^ { n } \int _ { - \tau ( \lambda ) } ^ { 0 } \left(y_{j}(t-(1-\alpha) \sigma+\theta)\right.\right. \\
& \left.\left.+x_{j}^{*}\right) d w_{i j}(\theta, \lambda)+I_{i}\right) \\
& -f_{i}\left(\sum_{j=1}^{n} \int_{-\tau(\lambda)}^{0} x_{j}^{*} d w_{i j}(\theta, \lambda)+I_{i}\right) .
\end{aligned}
$$


Substituting (19) into (12), we get

$$
\begin{aligned}
\frac{d y_{i}(t)}{d t}= & -a_{i}(\lambda) y_{i}(t)+\sigma a_{i}(\lambda) \\
& \times\left[-a_{i}(\lambda) y_{i}(t-(2-\alpha) \sigma)\right. \\
& +f_{i}\left(\sum _ { j = 1 } ^ { n } \int _ { - \tau ( \lambda ) } ^ { 0 } \left(y_{j}(t-(1-\alpha) \sigma+\theta)\right.\right. \\
& +f_{i}\left(\sum_{j=1}^{n} \int_{-\tau(\lambda)}^{0}\left(\sum_{j=1}^{n} \int_{-\tau(\lambda)}^{0} x_{j}^{*} d w_{i j}(\theta, \lambda)+I_{i}^{*}\right) d w_{i j}(\theta, \lambda)+I_{i}\right) \\
& -f_{i}\left(\sum_{j=1}^{n} \int_{-\tau(\lambda)}^{0} x_{j}^{*} d w_{i j}(\theta, \lambda)+I_{i}\right) .
\end{aligned}
$$
have

Let $V_{i}(t)=\left|y_{i}(t)\right|, i \in N$. Then, from $\left(\mathrm{A}_{1}\right)$ and $\left(\mathrm{A}_{2}\right)$, we

$$
\begin{aligned}
& D^{+} V_{i}(t) \\
& =\operatorname{sgn}\left(y_{i}(t)\right) \dot{y}_{i}(t) \\
& =\operatorname{sgn}\left(y_{i}(t)\right)\left\{-a_{i}(\lambda) y_{i}(t)+\sigma a_{i}(\lambda)\right. \\
& \times\left[-a_{i}(\lambda) y_{i}(t-(2-\alpha) \sigma)\right. \\
& +f_{i}\left(\sum _ { j = 1 } ^ { n } \int _ { - \tau ( \lambda ) } ^ { 0 } \left(y_{j}(t-(1-\alpha) \sigma+\theta)\right.\right. \\
& \left.\left.+x_{j}^{*}\right) d w_{i j}(\theta, \lambda)+I_{i}\right) \\
& \left.-f_{i}\left(\sum_{j=1}^{n} \int_{-\tau(\lambda)}^{0} x_{j}^{*} d w_{i j}(\theta, \lambda)+I_{i}\right)\right] \\
& +f_{i}\left(\sum _ { j = 1 } ^ { n } \int _ { - \tau ( \lambda ) } ^ { 0 } \left(y_{j}(t+\theta)\right.\right. \\
& \left.\left.+x_{j}^{*}\right) d w_{i j}(\theta, \lambda)+I_{i}\right) \\
& \left.-f_{i}\left(\sum_{j=1}^{n} \int_{-\tau(\lambda)}^{0} x_{j}^{*} d w_{i j}(\theta, \lambda)+I_{i}\right)\right\}
\end{aligned}
$$

$$
\begin{aligned}
\leq & -a_{i}(\lambda)\left|y_{i}(t)\right|+a_{i}^{2}(\lambda) \sigma\left[y_{i}(t)\right]_{r+(1-\alpha) \sigma}^{+} \\
& +a_{i}(\lambda) \sigma k_{i} \sum_{j=1}^{n} w_{i j}^{*}\left[y_{j}(t)\right]_{r+(1-\alpha) \sigma}^{+} \\
& +k_{i} \sum_{j=1}^{n} w_{i j}^{*}\left[y_{j}(t)\right]_{r+(1-\alpha) \sigma}^{+} \\
\leq & -\underline{a}_{i}\left|y_{i}(t)\right|+\sigma \bar{a}_{i}^{2}\left[y_{i}(t)\right]_{r+(1-\alpha) \sigma}^{+} \\
& +\left(\sigma \bar{a}_{i}+1\right) k_{i} \sum_{j=1}^{n} w_{i j}^{*}\left[y_{j}(t)\right]_{r+(1-\alpha) \sigma}^{+} \\
= & \sum_{j=1}^{n} u_{i j} V_{j}(t)+\sum_{j=1}^{n} v_{i j}\left[V_{j}(t)\right]_{r+(1-\alpha) \sigma}
\end{aligned}
$$

Since $-(U+V)$ is a nonsingular $M$-matrix, there exists a vector $z>0$ such that $(U+V) z<0$. By using the continuity, we know there exists at least one constant $\gamma>0$ such that

$$
\left(\gamma E+P+Q e^{\gamma(r+\sigma)}\right) z<0 .
$$

From (14) and (15), we know $y(t)$ is bounded on $[-r,(1-$ $\alpha) \sigma]$. So there exists a vector $\widehat{\eta}=\eta(1, \ldots, 1)^{T}$ such that $[y(t)]^{+} \leq \widehat{\eta}, t \in[-r,(1-\alpha) \sigma]$. Then we can get

$$
\begin{gathered}
v_{i}(t)=\left|y_{i}(t)\right| \leq \widehat{\eta} z e^{-\gamma(t-(1-\alpha) \sigma)}, \\
t \in[-r,(1-\alpha) \sigma], \quad i \in N .
\end{gathered}
$$

Then from (21), (22), (23), and Lemma 4 with $J=0$, we have

$$
\begin{gathered}
v_{i}(t)=\left|y_{i}(t)\right| \leq \widehat{\eta} z e^{-\gamma(t-(1-\alpha) \sigma)}, \\
t \in[(1-\alpha) \sigma, \infty], \quad i \in N .
\end{gathered}
$$

Let $M=z \hat{\eta} e^{\gamma \sigma}$, we get

$$
\left[x(t)-x^{*}\right]^{+} \leq M e^{-\gamma t}, \quad t \geq 0 .
$$

Thus, the equilibrium $x^{*}$ of system (1) is globally exponentially robustly stable.

Remark 7. If $\sigma=0$, the system (1) becomes the static interval neural networks without time delay in the leakage term. So, this paper includes the results of Han et al. (2011) as a special case.

Remark 8. If $\sigma=0$,

$$
\begin{gathered}
w_{i j}(\theta, \lambda)= \begin{cases}w_{i j}, & \theta=0, \\
0, & -\tau \leq \theta<0,\end{cases} \\
\tau(\lambda)=\tau, \quad a_{i}(\lambda)=a, \quad b_{i j}=\tau w_{i j},
\end{gathered}
$$

then system (1) becomes the following static neural network model:

$$
\begin{gathered}
\frac{d x_{i}(t)}{d t}=-a_{i} x_{i}(t)+f_{i}\left(\sum_{j=1}^{n} b_{i j} x_{j}(t)+I_{i}\right), \quad t \geq 0, \\
x_{i}(t)=\phi_{i}(t), \quad-r \leq t \leq 0 .
\end{gathered}
$$




$$
\begin{aligned}
& \text { If } \sigma=0 \text { and } \\
& \qquad w_{i j}(\theta, \lambda)= \begin{cases}\sum_{k=0}^{m} w_{i j}^{k}(\lambda), & \theta=\tau_{0}=0, \\
\sum_{k=1}^{m} w_{i j}^{k}(\lambda), & -\tau_{1} \leq \theta<0, \\
\sum_{k=2}^{m} w_{i j}^{k}(\lambda), & -\tau_{2} \leq \theta<-\tau_{1}, \\
\vdots & \\
w_{i j}^{m}(\lambda), & -\tau_{m} \leq \theta<-\tau_{m-1}, \\
0, & -\tau \leq \theta<\tau_{m},\end{cases}
\end{aligned}
$$

where $-\tau<-\tau_{m}<\cdots<-\tau_{1}<\tau_{0}=0$, then system (1) becomes a class of static neural network models with discrete time delays as follows:

$$
\begin{aligned}
\frac{d x_{i}(t)}{d t}= & -a_{i}(\lambda) x_{i}(t) \\
& +f_{i}\left(\sum_{k=0}^{m} \sum_{j=1}^{n} w_{i j}^{k}(\lambda) x_{j}\left(t-\tau_{k}\right)+I_{i}\right), \\
& t \geq 0, \\
& x_{i}(t)=\phi_{i}(t), \quad-r \leq t \leq 0 .
\end{aligned}
$$

If $\sigma=0$ and $w_{i j}(\theta, \lambda) \in C^{1}[-\tau, 0]$, then system (1) becomes the following static model with continuous time delays:

$$
\begin{gathered}
\frac{d x_{i}(t)}{d t}=-a_{i}(\lambda) x_{i}(t) \\
+f_{i}\left(\sum_{j=1}^{n} \int_{-\tau(\lambda)}^{0} x_{j}(t+\theta) w_{i j}^{\prime}(\theta, \lambda) d \theta+I_{i}\right), \\
x_{i}(t)=\phi_{i}(t), \quad-r \leq t \leq 0 .
\end{gathered}
$$

From (29) and (30), we can see that S-type distributed time delay contains discrete time delays and continuous time delays as two special cases, so our results generalized the results of the related literature $[1,3,10]$.

\section{Example}

Consider the following system:

$$
\begin{aligned}
\frac{d x_{1}(t)}{d t}= & -(2+\lambda) x_{1}\left(t-\frac{1}{10}\right) \\
& +\sin \left(\sum_{j=1}^{2} \int_{-\tau(\lambda)}^{0} x_{j}(t+\theta) d w_{i j}(\theta, \lambda)+1\right),
\end{aligned}
$$

$$
\begin{gathered}
\frac{d x_{2}(t)}{d t}=-(2+\lambda) x_{2}\left(t-\frac{1}{10}\right) \\
+\cos \left(\sum_{j=1}^{2} \int_{-\tau(\lambda)}^{0} x_{j}(t+\theta) d w_{i j}(\theta, \lambda)+2\right), \\
x_{i}(t)=\phi_{i}(t), \quad-r \leq t \leq 0,
\end{gathered}
$$

where $\lambda \in[0,1],\left|\int_{-\tau(\lambda)}^{0} d w_{11}(\theta, \lambda)\right| \leq 1 / 3$, $\left|\int_{-\tau(\lambda)}^{0} d w_{12}(\theta, \lambda)\right| \leq 1 / 2,\left|\int_{-\tau(\lambda)}^{0} d w_{21}(\theta, \lambda)\right| \leq 1 / 2$, and $\left|\int_{-\tau(\lambda)}^{0} d w_{22}(\theta, \lambda)\right| \leq 1 / 4, k_{1}=k_{2}=1$. It can be obtained that

$$
U=\left(\begin{array}{cc}
-2 & 0 \\
0 & -2
\end{array}\right), \quad V=\left(\begin{array}{cc}
\frac{4}{3} & \frac{13}{20} \\
\frac{13}{20} & \frac{49}{40}
\end{array}\right) .
$$

Thus

$$
-(U+V)=-\left(\begin{array}{rr}
-\frac{2}{3} & \frac{13}{20} \\
\frac{13}{20} & -\frac{31}{40}
\end{array}\right)
$$

is a $M$-matrix. From Theorem 6 , the equilibrium point of system (27) is globally exponentially robust stable.

\section{Conflict of Interests}

The authors declare that there is no conflict of interests regarding the publication of this paper.

\section{Acknowledgments}

The work was supported by the National Natural Science Foundation of China (11171374) and Natural Science Foundation of Shandong Province (ZR2011AZ001).

\section{References}

[1] Z. Wu, J. Lam, H. Su et al., "Stability and dissipativity analysis of static neural networks with time delay," IEEE Transactions on Neural Networks and Learning Systems, vol. 23, no. 2, pp. 199210, 2012.

[2] L. Wang, R. Zhang, and Y. Wang, "Global exponential stability of reaction-diffusion cellular neural networks with Stype distributed time delays," Nonlinear Analysis: Real World Applications, vol. 10, no. 2, pp. 1101-1113, 2009.

[3] Q. Duan, H. Su, and Z. Wu, " $H_{\infty}$ state estimation of static neural networks with time-varying delay," Neurocomputing, vol. 97, no. 15, pp. 16-21, 2012.

[4] L. Wan and D. Xu, "Global exponential stability of Hopfield reaction-diffusion neural networks with time-varying delays," Science in China F, vol. 46, no. 6, pp. 466-474, 2003.

[5] D. Xu and S. Long, "Attracting and quasi-invariant sets of nonautonomous neural networks with delays," Neurocomputing, vol. 77, no. 1, pp. 222-228, 2012. 
[6] L. Wang and D. Xu, "Global asymptotic stability of bidirectional associative memory neural networks with S-type distributed delays," International Journal of Systems Science, vol. 33, no. 11, pp. 869-877, 2002.

[7] L. Wang, Recurrent Neural Networks with Time Delays, Science Press, Beijing, China, 2008.

[8] C. Huang and J. Cao, "Almost sure exponential stability of stochastic cellular neural networks with unbounded distributed delays," Neurocomputing, vol. 72, no. 13-15, pp. 3352-3356, 2009.

[9] Z.-G. Wu, J. H. Park, H. Su, and J. Chu, "New results on exponential passivity of neural networks with time-varying delays," Nonlinear Analysis: Real World Applications, vol. 13, no. 4, pp. 1593-1599, 2012.

[10] W. Han, Y. Kao, and L. Wang, "Global exponential robust stability of static interval neural networks with S-type distributed delays," Journal of the Franklin Institute, vol. 348, no. 8, pp. 20722081, 2011.

[11] K. Gopalsamy, Stability and Oscillations in Delay Differential Equations of Population Dynamics, vol. 74, Kluwer Academic, Dordrecht, The Netherlands, 1992.

[12] X. Li, R. Rakkiyappan, and P. Balasubramaniam, "Existence and global stability analysis of equilibrium of fuzzy cellular neural networks with time delay in the leakage term under impulsive perturbations," Journal of the Franklin Institute, vol. 348, no. 2, pp. 135-155, 2011.

[13] K. Gopalsamy, "Leakage delays in BAM," Journal of Mathematical Analysis and Applications, vol. 325, no. 2, pp. 1117-1132, 2007.

[14] C. Li and T. Huang, "On the stability of nonlinear systems with leakage delay," Journal of the Franklin Institute, vol. 346, no. 4, pp. 366-377, 2009.

[15] X. Li, X. Fu, P. Balasubramaniam, and R. Rakkiyappan, "Existence, uniqueness and stability analysis of recurrent neural networks with time delay in the leakage term under impulsive perturbations," Nonlinear Analysis: Real World Applications, vol. 11, no. 5, pp. 4092-4108, 2010.

[16] M. J. Park, O. M. Kwon, J. H. Park, S. M. Lee, and E. J. Cha, "Synchronization criteria for coupled stochastic neural networks with time-varying delays and leakage delay," Journal of the Franklin Institute, vol. 349, no. 5, pp. 1699-1720, 2012.

[17] S. Peng, "Global attractive periodic solutions of BAM neural networks with continuously distributed delays in the leakage terms," Nonlinear Analysis: Real World Applications, vol. 11, no. 3, pp. 2141-2151, 2010.

[18] X. Li, R. Rakkiyappan, and P. Balasubramaniam, "Existence and global stability analysis of equilibrium of fuzzy cellular neural networks with time delay in the leakage term under impulsive perturbations," Journal of the Franklin Institute, vol. 348, no. 2, pp. 135-155, 2011.

[19] A. Berman and R. J. Plemmons, Nonnegative Matrices in the Mathematical Sciences, Academic Press, New York, NY, USA, 1979.

[20] D. Xu and Z. Yang, "Attracting and invariant sets for a class of impulsive functional differential equations," Journal of Mathematical Analysis and Applications, vol. 329, no. 2, pp. 1036-1044, 2007. 


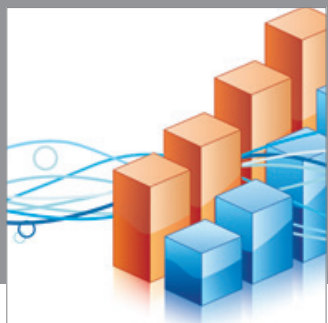

Advances in

Operations Research

mansans

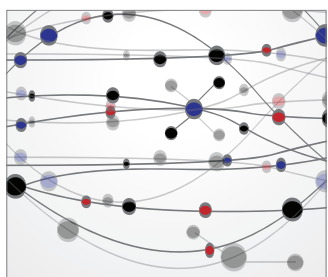

The Scientific World Journal
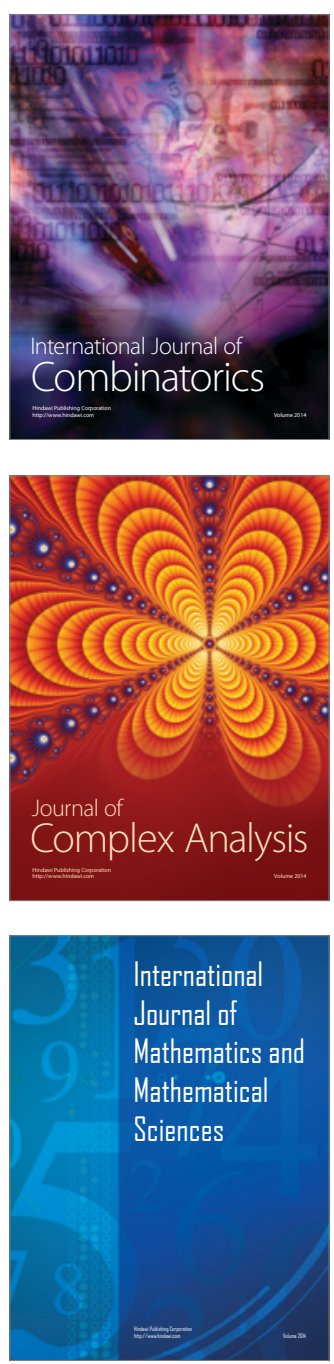
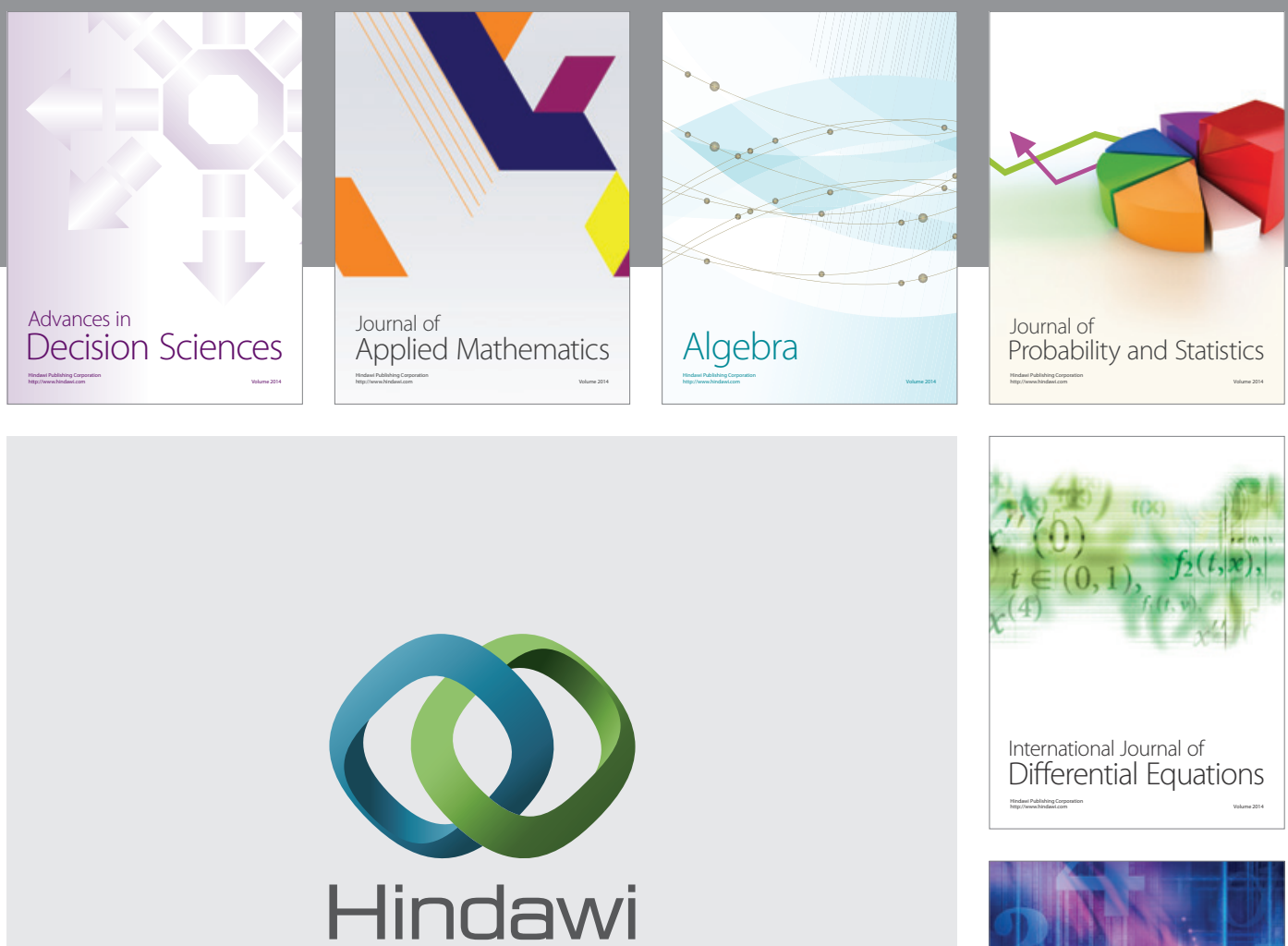

Submit your manuscripts at http://www.hindawi.com
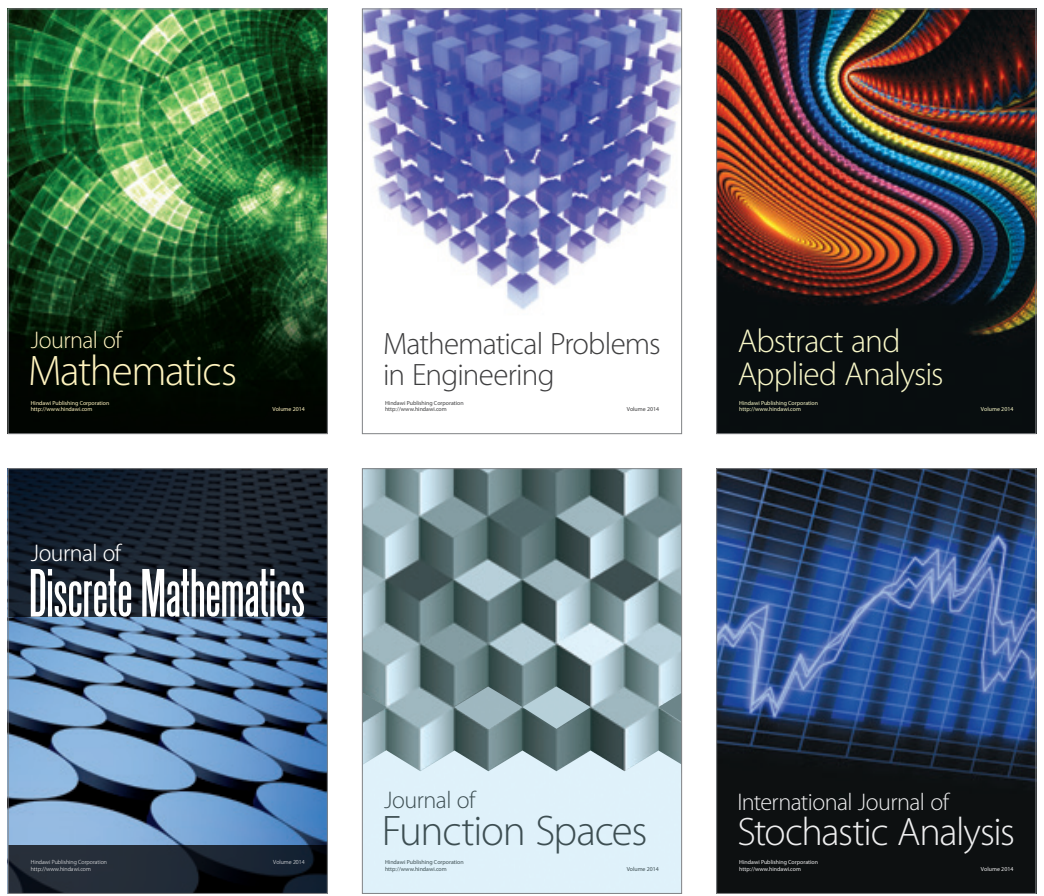

Journal of

Function Spaces

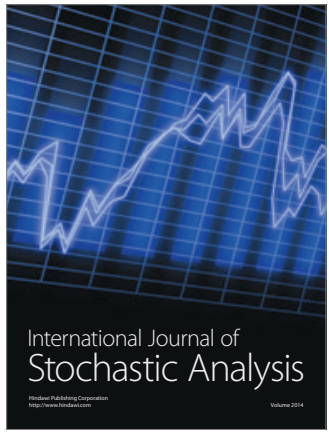

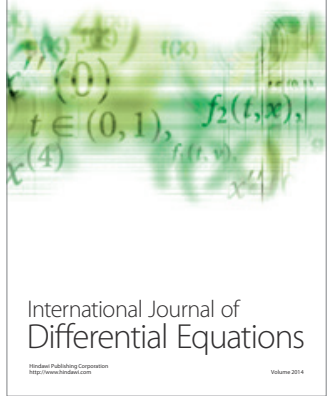
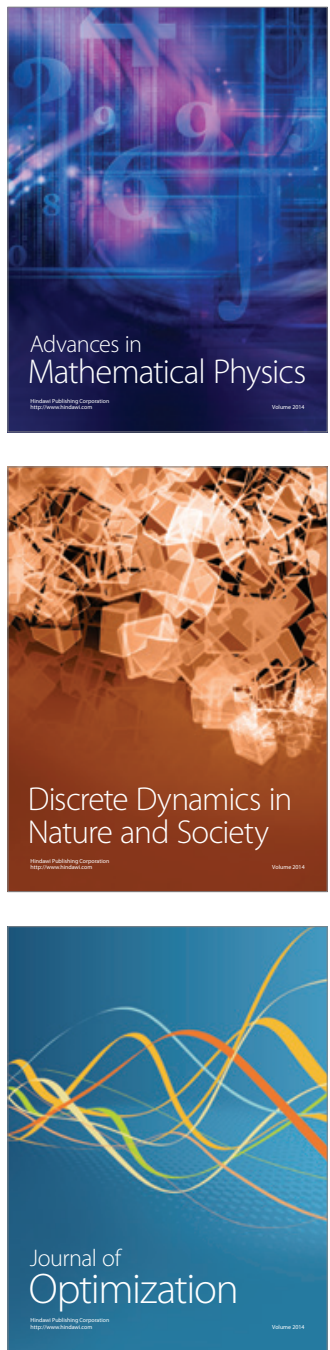